\title{
Calibration of the RPC charge readout in the ARGO-YBJ experiment
}

\author{
G. Aielli ${ }^{\text {a,b }}$, C. Bacci ${ }^{\text {c,d }}$, B. Bartoli ${ }^{\text {e,f }}$, P. Bernardini ${ }^{\text {g,h }}$, X.J. Bi ${ }^{\text {i }}$, C. Bleve ${ }^{\text {g,h }}$, P. Branchini ${ }^{\text {d }}$, A. Budano ${ }^{\text {, }}$,
} S. Bussino ${ }^{\text {c,d }}$, A.K. Calabrese Melcarne ${ }^{\mathrm{j}}$, P. Camarri ${ }^{\mathrm{a}, \mathrm{b}}$, Z. Cao ${ }^{\mathrm{i}}$, A. Cappa ${ }^{\mathrm{k}, \mathrm{l}}$, R. Cardarelli ${ }^{\mathrm{b}}$, S. Catalanotti ${ }^{\mathrm{e}, \mathrm{f}}$, C. Cattaneo $^{\mathrm{m}}$, P. Celio ${ }^{\mathrm{c}, \mathrm{d}}$, S.Z. Chen ${ }^{\mathrm{i}}$, T.L. Chen ${ }^{\mathrm{s}}$, Y. Chen ${ }^{\mathrm{i}}$, N. Cheng ${ }^{\mathrm{i}}$, P. Creti $^{\mathrm{h}}$, S.W. Cui ${ }^{\text {}}$, B.Z. Dai ${ }^{\mathrm{p}}$, G. D’Alí Staiti ${ }^{\mathrm{r}, \mathrm{q}}$, Danzengluobu ${ }^{\mathrm{s}}$, M. Dattoli ${ }^{\mathrm{k}, \mathrm{l}, \mathrm{t}}$, I. De Mitri ${ }^{\mathrm{g}, \mathrm{h}}$, B. D’Ettorre Piazzoli ${ }^{\text {e,f }}$, M. De Vincenzi $^{\mathrm{c}, \mathrm{d}}$, T. Di Girolamo ${ }^{\text {e,f }}$, X.H. Ding ${ }^{\text {s }, ~ G . ~ D i ~ S c i a s c i o ~}{ }^{\text {b }}$, C.F. Feng ${ }^{\mathrm{u}}$, Zhaoyang Feng ${ }^{\mathrm{i}}$, Zhenyong Feng ${ }^{\mathrm{v}}$, F. Galeazzi ${ }^{\mathrm{d}}$, P. Galeotti ${ }^{\text {k,t }}$, R. Gargana ${ }^{d}$, Q.B. Gou ${ }^{\mathrm{i}}$, Y.Q. Guo ${ }^{\mathrm{i}}$, H.H. He ${ }^{\mathrm{i}}$, Haibing Hu ${ }^{\mathrm{s}}$, Hongbo Hu ${ }^{\mathrm{i}}$, Q. Huang ${ }^{\mathrm{v}}$, M. Iacovacci ${ }^{\text {e,f }}$, R. Iuppa ${ }^{\text {a,b }}$, I. James ${ }^{\text {c,d }}$, H.Y. Jia ${ }^{\text {v }}$, Labaciren ${ }^{\text {s }}$, H.J. Li ${ }^{\text {s }}$, J.Y. Li ${ }^{\text {u }}$, X.X. Li ${ }^{\text {i,* }}$, B. Liberti ${ }^{b}$, G. Liguori ${ }^{\mathrm{m}, \mathrm{n}}$, C. Liu $^{\mathrm{i}}$, C.Q. Liu ${ }^{\mathrm{p}}$, M.Y. Liu $^{\mathrm{o}}$, J. Liu ${ }^{\mathrm{p}}$, H. Lu ${ }^{\mathrm{i}}$, X.H. Ma ${ }^{\mathrm{i}}$, G. Mancarella ${ }^{\mathrm{g}, \mathrm{h}}$, S.M. Mari $^{\mathrm{c}, \mathrm{d}}$, G. Marsella ${ }^{\text {w,h }}$, D. Martello ${ }^{\text {g,h }}$, S. Mastroianni ${ }^{\text {, }}$ X.R. Meng ${ }^{\text {s }}$, P. Montini ${ }^{\text {c,d }}$, C.C. Ning ${ }^{\text {s }}$, A. Pagliaro ${ }^{\mathrm{x}, \mathrm{r}}$, M. Panareo ${ }^{\text {w,h }}$, L. Perrone ${ }^{\text {w,h }}$, P. Pistilli ${ }^{\text {c,d }}$, X.B. Qu ${ }^{\text {u, E. Rossi }}{ }^{\text {f }}$, F. Ruggieri ${ }^{\text {d }}$, L. Saggese ${ }^{\text {e,f }}$, P. Salvini ${ }^{\mathrm{m}}$, R. Santonico ${ }^{\mathrm{a}, \mathrm{b}}$, P.R. Shen ${ }^{\mathrm{i}}$, X.D. Sheng ${ }^{\mathrm{i}}$, F. Shi ${ }^{\mathrm{i}}$, C. Stanescu ${ }^{\mathrm{d}}$, A. Surdo ${ }^{\text {h }}$, Y.H. Tan ${ }^{\mathrm{i}}$, P. Vallania ${ }^{\mathrm{k}, 1}$, S. Vernetto ${ }^{k, 1}$, C. Vigorito ${ }^{\mathrm{k}, 1}$, B. Wang ${ }^{\mathrm{i}}$, H. Wang ${ }^{\mathrm{i}}$, C.Y. Wu ${ }^{\mathrm{i}}$, H.R. Wu ${ }^{\mathrm{i}}$, B. Xu ${ }^{\mathrm{v}}$, L. Xue ${ }^{\mathrm{u}}$, Y.X. Yan ${ }^{\mathrm{o}}$, Q.Y. Yang ${ }^{p}$, X.C. Yang ${ }^{p}$, A.F. Yuan ${ }^{\mathrm{s}}$, M. Zha ${ }^{\mathrm{i}}$, H.M. Zhang ${ }^{\mathrm{i}}$, JiLong Zhang ${ }^{\mathrm{i}}$, JianLi Zhang ${ }^{\mathrm{i}}$, L. Zhang ${ }^{\mathrm{p}}$, P. Zhang ${ }^{\text {, }}$ X.Y. Zhang ${ }^{\mathrm{u}}$, Y. Zhang ${ }^{\mathrm{i}}$, Zhaxisangzhu ${ }^{\text {s }}$, X.X. Zhou ${ }^{\text {v }}$, F.R. Zhu ${ }^{\mathrm{i}}$, Q.Q. Zhu ${ }^{\mathrm{i}}$, G. Zizzi ${ }^{\text {g,h }}$

a Dipartimento di Fisica dell'Università “Tor Vergata”, via della Ricerca Scientifica 1, 00133 Roma, Italy

${ }^{\mathrm{b}}$ Istituto Nazionale di Fisica Nucleare, Sezione di Tor Vergata, via della Ricerca Scientifica 1, 00133 Roma, Italy

'Dipartimento di Fisica dell'Università "Roma Tre", via della Vasca Navale 84, 00146 Roma, Italy

d Istituto Nazionale di Fisica Nucleare, Sezione di Roma3, via della Vasca Navale 84, 00146 Roma, Italy

e Dipartimento di Fisica dell'Università di Napoli, Complesso Universitario di Monte Sant'Angelo, via Cintia, 80126 Napoli, Italy

${ }^{\mathrm{f}}$ Istituto Nazionale di Fisica Nucleare, Sezione di Napoli, Complesso Universitario di Monte Sant'Angelo, via Cintia, 80126 Napoli, Italy

${ }^{g}$ Dipartimento di Fisica dell'Università del Salento, via per Arnesano, 73100 Lecce, Italy

h Istituto Nazionale di Fisica Nucleare, Sezione di Lecce, via per Arnesano, 73100 Lecce, Italy

${ }^{\mathrm{i}}$ Key Laboratory of Particle Astrophyics, Institute of High Energy Physics, Chinese Academy of Science, P.O. Box 918, 100049 Beijing, PR China

${ }^{\mathrm{j}}$ Istituto Nazionale di Fisica Nucleare, CNAF, viale Berti-Pichat 6/2, 40127 Bologna, Italy

${ }^{\mathrm{k}}$ Istituto Nazionale di Fisica Nucleare, Sezione di Torino, via P. Giuria 1, 10125 Torino, Italy

${ }^{1}$ Istituto di Fisica dello Spazio Interplanetario dell'Istituto Nazionale di Astrofisica, corso Fiume 4, 10133 Torino, Italy

m Istituto Nazionale di Fisica Nucleare, Sezione di Pavia, via Bassi 6, 27100 Pavia, Italy

${ }^{\mathrm{n}}$ Dipartimento di Fisica Nucleare e Teorica dell'Università di Pavia, via Bassi 6, 27100 Pavia, Italy

${ }^{\circ}$ Hebei Normal University, Shijiazhuang 050016, Hebei, China

p Yunnan University, 2 North Cuihu Rd, 650091 Kunming, Yunnan, PR China

${ }^{\mathrm{q}}$ Università degli Studi di Palermo, Dipartimento di Fisica e Tecnologie Relative, Viale delle Scienze, Edificio 18, 90128 Palermo, Italy

${ }^{\mathrm{r}}$ Istituto Nazionale di Fisica Nucleare, Sezione di Catania, Viale A. Doria 6, 95125 Catania, Italy

s Tibet University, 850000 Lhasa, Xizang, PR China

${ }^{\mathrm{t}}$ Dipartimento di Fisica Generale dell'Università di Torino, via P. Giuria 1, 10125 Torino, Italy

" Shandong University, 250100 Jinan, Shandong, PR China

${ }^{v}$ South West Jiaotong University, 610031 Chengdu, Sichuan, PR China

${ }^{\mathrm{w}}$ Dipartimento di Ingegneria dell'Innovazione, Università del Salento, 73100 Lecce, Italy

${ }^{\mathrm{x}}$ Istituto di Astrofisica Spaziale e Fisica Cosmica di Palermo, Istituto Nazionale di Astrofisica, via Ugo La Malfa 153, 90146 Palermo, Italy

\section{A R T I C L E I N F O}

\section{Available online 30 September 2010}

\section{Keywords:}

Resistive plate chambers

Charge readout

Calibration

\begin{abstract}
A B S T R A C T
The charge readout of Resistive Plate Chambers (RPCs) is implemented in the ARGO-YBJ experiment to measure the charged particle density of the shower front up to $10^{4} / \mathrm{m}^{2}$, enabling the study of the primary cosmic rays with energies in the "knee" region. As the first time for RPCs being used this way, a telescope with RPCs and scintillation detectors is setup to calibrate the number of charged particles hitting a RPC versus its charge readout. Air shower particles are taken as the calibration beam.
\end{abstract}

\footnotetext{
* Corresponding author. Tel.: +861088236106; fax: +861088233086

E-mail address: lixx@ihep.ac.cn (X.X. Li).
} 
The telescope was tested at sea level and then moved to the ARGO-YBJ site for coincident operation with the ARGO-YBJ experiment. The charge readout shows good linearity with the particle density in the dynamic range (up to $200 / \mathrm{m}^{2}$ ).

(c) 2010 Elsevier B.V. All rights reserved.

\section{Introduction}

The ARGO-YBJ experiment [1], located at Yangbajing Cosmic Ray Observatory (Tibet, PR China, $4300 \mathrm{~m}$ a.s.l.), consists of a single layer of RPCs operated in streamer mode. Each RPC $\left(2.8 \times 1.25 \mathrm{~m}^{2}\right)$ is read by 80 strips of $6.75 \times 61.8 \mathrm{~cm}^{2}$, logically organized in 10 independent pads of $55.6 \times 61.8 \mathrm{~cm}^{2}$ (digital readout). Twelve RPCs are grouped into a so-called cluster $\left(5.7 \times 7.6 \mathrm{~m}^{2}\right)$. The central part of the detector, $5600 \mathrm{~m}^{2}$ in area, is fully covered by 130 clusters, while 23 clusters surrounding the central carpet form a guard ring. The whole array covers a total area of about $10,000 \mathrm{~m}^{2}$.

As one of the main physics goals, the ARGO-YBJ experiment will study the "knee" of the cosmic ray spectrum by precisely measuring cosmic ray showers above few $\mathrm{TeV}$ up to the $\mathrm{PeV}$ region. This requires a measurement of the particle density from $0.1 / \mathrm{m}^{2}$ to $10^{4} / \mathrm{m}^{2}[2]$. With a strip density of about $22 \mathrm{strips} / \mathrm{m}^{2}$, the digital readout provides a linear response up to a shower core particle density of about $15 / \mathrm{m}^{2}$, corresponding to a primary energy of $200 \mathrm{TeV}$. To measure the particle density up to $10^{4} / \mathrm{m}^{2}$, a charge readout is implemented by instrumenting every RPC with two large size pads of dimension $140 \times 125 \mathrm{~cm}^{2}$ each, the so-called "Big Pads". The electronic unit to read out the charge signals is a MINICRATE that has two sections, each one hosting three readout cards and one control board serving for one cluster [3-6].

The charge readout to measure particle density has never been performed on RPCs before. This is the first time for RPCs being used this way in Extensive Air Shower (EAS) experiments at high altitude. The behavior of the RPC charge readout cannot be foreseen at such a high particle density. For a gaseous detector working at high altitude, a calibration at the same site is strongly recommended. A dedicated telescope has been installed in the Yangbajing Cosmic Ray Observatory for calibration purposes. In this paper we describe the experimental set-up and the procedure applied to determine the RPC charge output as a function of the particle number.

\section{Experiment setup and data taking}

The key points in this calibration lie on the availability of charged particle beams and the determination of the exact number of charged particles impinging on the RPC to be calibrated. To perform the calibration of the ARGO-YBJ RPCs, a telescope (Fig. 1) is setup with two scintillation detectors, to measure the number of charged particles impinging on them, and five RPCs. The RPC (RPC3) between the two scintillation detectors acts as the one to be calibrated. Two RPCs (RPC2 and RPC4) provide pad and strip information and can also be used to check the calibration. RPCO and RPC1 help to pick up coincident events from ARGO-YBJ data. Charged particles of EASs are taken as the calibration beam.

Each scintillation detector of size of $275 \mathrm{~cm} \times 125 \mathrm{~cm}$ consists of $5 \times 11$ so-called tiles, $25 \mathrm{~cm} \times 25 \mathrm{~cm} \times 2 \mathrm{~cm}$ each, covering the dimension of one RPC (the difference is less than $2 \%$, which can be corrected). Light due to energy loss by charged particles in a tile is collected by eight single cladding fibers glued into grooves at the tile surface. A total of 440 fibers from one scintillation detector is coped with one photomultiplier tube (PMT).

As in the ARGO-YBJ experiment, the RPCs are operated at a high voltage of $7200 \mathrm{~V}$ with a gas mixture of Argon (15\%), tetrafluoroethane (R134A, 75\%) and isobutane (10\%). The efficiency is greater than $96 \%$ [7].

The digital readouts of all the five RPCs in the telescope are merged into the ARGO-YBJ data acquisition system (DAQ) with the telescope acting as a normal cluster. Furthermore dedicated electronics and a DAQ independent of the ARGO-YBJ experiment are designed for the calibration. Charge readout signals from Big Pads of RPC2-4 and signals from the scintillation detectors are filtered and shaped, then they are split into two channels with, respectively, low gain (LG) and high gain (HG) amplifiers, achieving a dynamic range of 3.5 orders of magnitude. Signals from each HG/LG amplifier are digitized by a Flash Analog-toDigital-Converter (FADC, 10 bits, $50 \mathrm{MHz}$ ). A Field Programmable Gate Array (FPGA) collects and analyzes the digital data from FADCs. A channel is fired if the amplitude exceeds the preset threshold. If RPC3 and both scintillation detectors are fired, a trigger is generated and the digitized pulse shape data are pushed into a buffer in the FPGA. An embedded computer polls the buffer through its PC104 bus and transfers the data when valid. On receiving a trigger, a GPS-based timing system will record the event time with a precision of better than $100 \mathrm{~ns}$ for purpose of off-line coincidence with the ARGO-YBJ experiment.

To cover the whole needed dynamic range of 4 orders of magnitude, the calibration is done in two steps: below 200 particles $/ \mathrm{m}^{2}$ and above 100 particles $/ \mathrm{m}^{2}$, with the PMTs working at different High Voltages (HVs), 500 and $400 \mathrm{~V}$, respectively. The linearities and HV responses of PMTs and amplifier gains were calibrated in detail. The HV responses of PMTs show a nonlinearity less than $1 \%$ from 350 to $750 \mathrm{~V}$. The nonlinearities of PMTs are less than $5 \%$ in 2.5 orders of magnitude.

After tested and optimized at sea level, the telescope was moved to Yangbajing and installed in the guard ring of the ARGO-YBJ experiment, to perform coincident observation of air showers with the ARGO-YBJ experiment.

\section{Data analysis and results}

A match between the telescope events and the ARGO-YBJ ones in a time window of $1 \mu \mathrm{s}$ is performed off-line based on the event time. In case of a match, ARGO-YBJ data provide general information of an air shower such as fired pads and strips, reconstructed

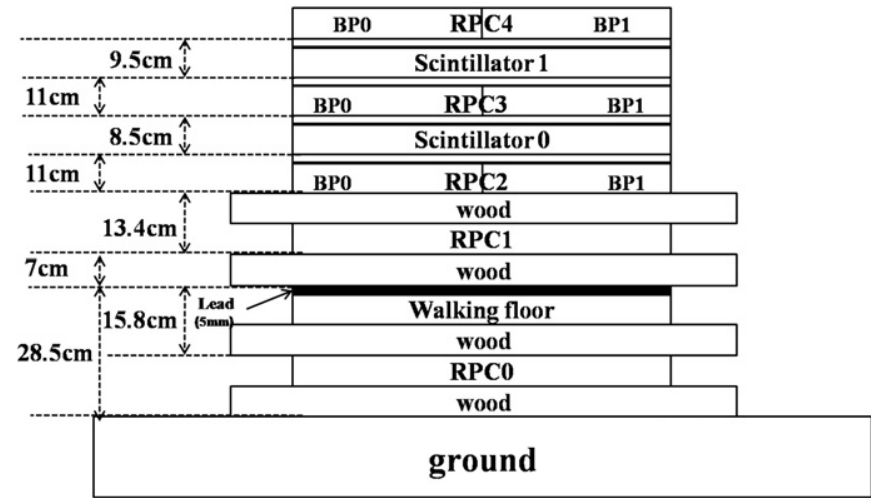

Fig. 1. Sketch of the calibration telescope. 
primary direction and core location, etc. Only matched events are used in the following data analysis. The measured amplitudes of scintillation detectors of the telescope are corrected according to the above mentioned calibration results.

When charged particles pass through a RPC, charges are induced in both Big Pads, i.e. the total induced charges are shared by the two Big Pads. In the following analysis, the charges of the two Big Pads in one RPC are summed as the total charge induced in this RPC.

\subsection{Amplitude spectra induced by a single particle}

Events with only one particle passing through the telescope are picked up by using the pad information in digital readout, i.e. only one pad is fired in each of the 3 RPCs (RPC2, 3, 4). The amplitude distribution of one scintillation detector induced by a single particle is shown in Fig. 2, which approximately follows a Landau distribution.

The distribution of charge readout of one RPC in case of a single particle impinging on it is shown in Fig. 3, where the first peak corresponds to cases with only one streamer generated in the gas chamber (single streamer peak), while the tail of the distribution shows that multiple streamers are generated when only one particle passes through the gas volume. The single streamer peak follows a Gaussian distribution and contributes $90 \%$ of all signals with a resolution of about $14 \%$, while the single particle resolution is worse due to the contribution of multiple streamers.

\subsection{Absolute calibration of RPC charge readout}

Since air shower particles are treated as the calibration beam, the number of triggered events decreases with increasing secondary particle density. In the analysis the RPC charges are binned with increasing bin width for statistical convenience. For each RPC charge bin the average number of particles is predicted by the corresponding distribution provided by the scintillation counter. The particle density region $>20 / \mathrm{m}^{2}$, where saturation effects prevent the use of the digital readout, has been carefully investigated. In this case the signal amplitude of the scintillation detector follows, according to the Central Limit Theorem,

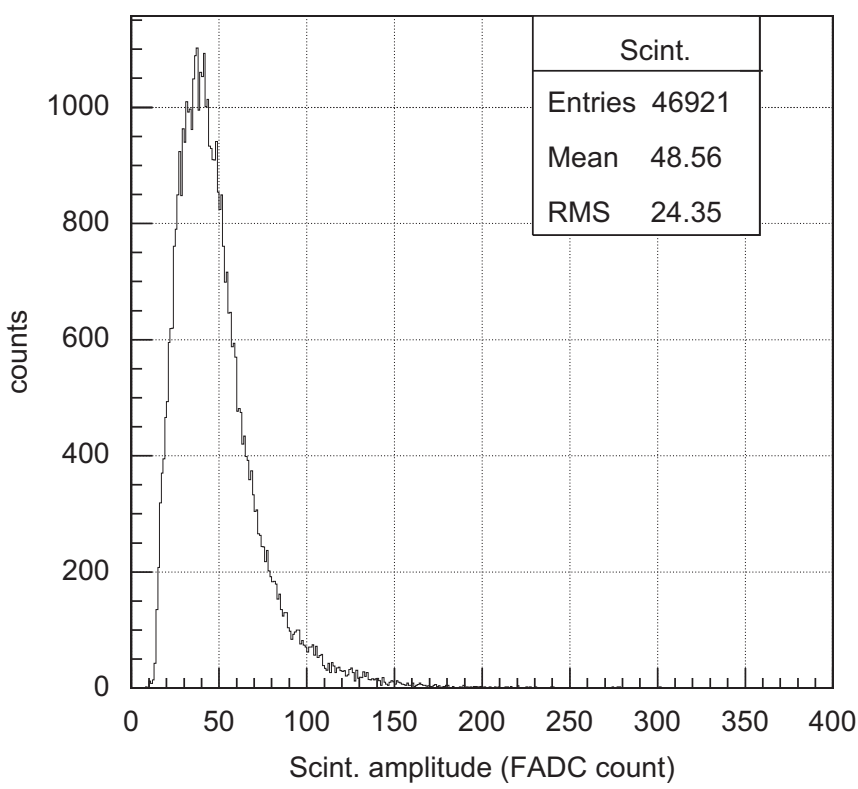

Fig. 2. Amplitude distribution of one scintillation detector in case of a single particle.

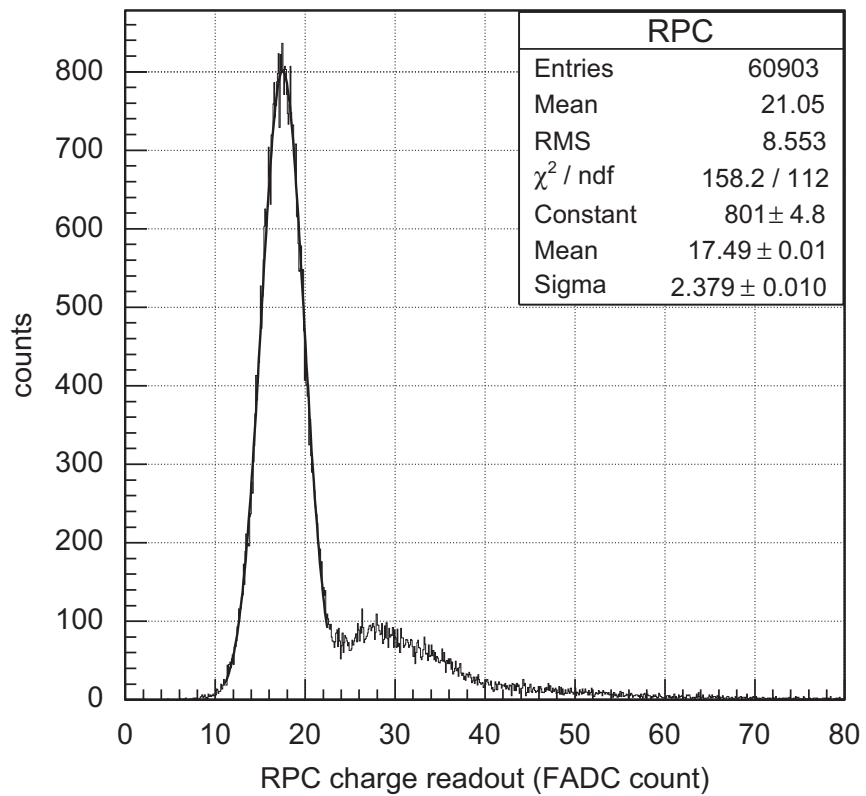

Fig. 3. Charge readout of one RPC in case of single particle. The black curve presents a Gaussian fit to the single streamer peak.

a Gaussian distribution, allowing to determine the average number of particles and its relative uncertainty.

With data collected in about 10 days enough statistical accuracy ( $3 \%$ relative error) up to particle densities of about $200 / \mathrm{m}^{2}$ has been achieved. The relation between the predicted number of particles and the RPC charge output is shown in Fig. 4(left). By a linear fit to these data an average charge readout of 20.8 FADC counts per particle is obtained, consistent with the mean value of the RPC single particle spectrum (Fig. 3). A residual nonlinearity $<6 \%$ is found and shown in Fig. 4(right) as a function of the number of particles crossing the RPC. This nonlinearity results from the combined effect of many causes as, for instance, the intrinsic nonlinearity of the RPC, the dependence of the RPC response on pressure, temperature and humidity, the nonlinearity of PMTs and electronics, the uncertainty on the particle number predictions.

As the first step of the calibration, the dynamic range below 200 particles $/ \mathrm{m}^{2}$ is considered. At higher particle densities the statistics is still poor due to the dead time of the telescope DAQ caused by the high trigger rate of low particle density events. In the next step, the single channel thresholds will be increased to decrease the event rate thus decreasing the dead time. That promises the needed statistics at high particle densities.

It should be pointed out that some shower particles with low energy may stop before reaching RPC3. Furthermore for high particle density events, i.e. events with high primary energies, there exist abundant high energy shower particles, including many photons of energy $>1 \mathrm{MeV}$, that have chance to react and generate more charged particles as traversing the telescope. The number of particles traversing RPC3 might be slightly different from that measured by either scintillation detector. Since RPC3 is sandwiched between the two scintillation detectors, the average over the measurements of the two scintillation detectors can be a better estimate to the number of particles traversing RPC3. Studies on this are still undergoing.

\section{Conclusions}

A telescope of scintillation counters and RPCs has been installed inside the ARGO-YBJ array to study the RPC charge output as a function of the number of charged particles hitting the detector. The approach implemented for this study exploits the coincident 

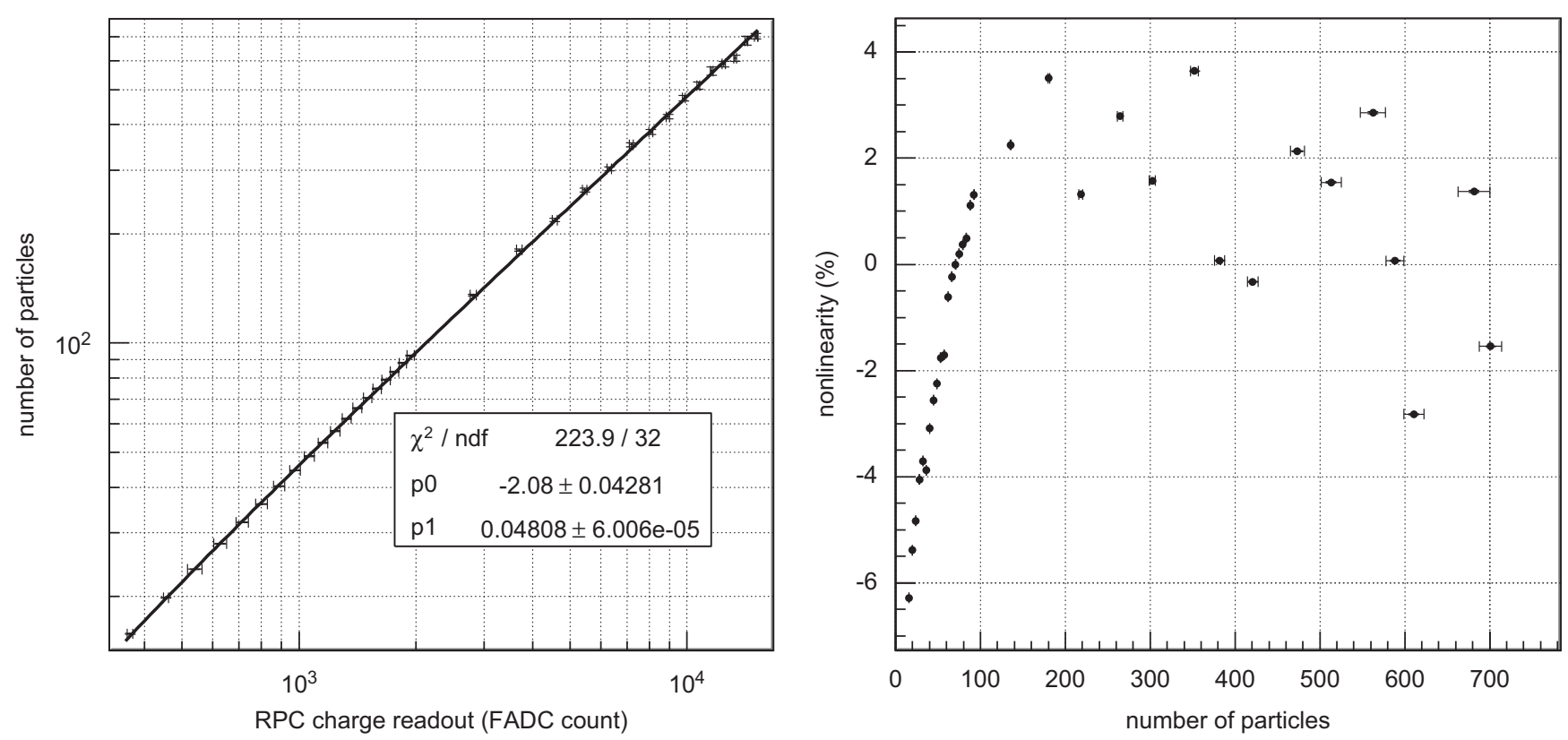

Fig. 4. Number of charged particles versus the RPC charge readout with a linear fit (left) and nonlinearity of the calibration (right).

measurement of air showers with the ARGO-YBJ experiment to select events with high particle density. Ten days of data taking allowed a preliminary measurement of the RPC response up to a density of about 200 particles $/ \mathrm{m}^{2}$. No saturation effects have been observed. The RPC charge output exhibits a good linearity, the residual nonlinearity being less than 6\%. Many effects may contribute to this figure. In order to determine the intrinsic linearity of the RPCs operated at Yangbajing, a more detailed analysis is required aimed to disentangle the other contributions. The measurement of the RPC response to higher particle densities up to 10,000 particles $/ \mathrm{m}^{2}$ needs a long data taking by means of an inclusive trigger selecting large size showers. This topic will be addressed in a future work.

\section{Acknowledgements}

This work is supported in China by NSFC (10120130794), the Chinese Ministry of Science and Technology, the Chinese Academy of Sciences, the Key Laboratory of Particle Astrophysics, CAS, and in Italy by the Istituto Nazionale di Fisica Nucleare (INFN).

We also acknowledge the essential supports of W.Y. Chen, G. Yang, X.F. Yuan, C.Y. Zhao, R. Assiro, B. Biondo, S. Bricola, F. Budano, A. Corvaglia, B. D’Aquino, R. Esposito, A. Innocente, A. Mangano, E. Pastori, C. Pinto, E. Reali, F. Taurino and A. Zerbini, in the installation, debugging and maintenance of the detector.

\section{References}

[1] G. Aielli, et al., Nucl. Instr. and Meth. A 562 (2006) 92.

[2] M. Iacovacci, et al., Nucl. Phys. B 136 (2004) 376.

[3] P. Creti, et al., in: 29th ICRC, vol. 8, Pune, 2005, pp. 97-100.

[4] M. Iacovacci, et al., in: 28th ICRC, vol. 757, Tsukuba, 2003.

[5] Q.Y. YANG, et al., HEP \& NP 28 (8) (2004) 866.

[6] A. Corvaglia, et al., in: 31st ICRC, Lodz, 2009.

[7] G. Aielli, et al., Nucl. Instr. and Meth. A 608 (2009) 246. 